\title{
CORRECTION
}

Hao Fu $\cdot$ Chuangxin He ${ }_{(i)} \cdot$ Yingzheng Liu

\section{Correction to: Flow structures of a precessing jet in an axisymmetric chamber}

Published online: 22 April 2021

(C) The Visualization Society of Japan 2021

\section{Correction to: J Vis \\ https://doi.org/10.1007/s12650-020-00722-2}

In the original paper (Fu et al. 2021), we made a mistake on the determination of the bulk inflow velocity $U_{0} . U_{0} \approx 1.12 \mathrm{~m} / \mathrm{s}$ instead of $0.60 \mathrm{~m} / \mathrm{s}$ in the original paper. Thus, all the normalizations with respect to $U_{0}$ in the related figures and texts should be corrected. The Reynolds number reported in the original paper should be corrected to $4.5 \times 10^{4}$, and the two Strouhal numbers, St $\approx 7.4 \times 10^{-3}$ and St $\approx 6.0 \times 10^{-3}$ reported in the article, should be revised to $\mathrm{St} \approx 4.0 \times 10^{-3}$ and $\mathrm{St} \approx 3.2 \times 10^{-3}$, respectively. The contour legends and the abscissas of the related figures in which the normalization with respect to $U_{0}$ was used should be scaled accordingly. We confirm that these corrections do not affect the discussion and conclusions of the original paper.

\section{Reference}

Fu H, He C, Liu Y (2021) Flow structures of a precessing jet in an axisymmetric chamber. J Vis. https://doi.org/10.1007/ s12650-020-00722-2

Publisher's Note Springer Nature remains neutral with regard to jurisdictional claims in published maps and institutional affiliations.

The original article can be found online at https://doi.org/10.1007/s12650-020-00722-2.

H. Fu · C. He $(\bowtie) \cdot$ Y. Liu

Key Lab of Education Ministry for Power Machinery and Engineering, School of Mechanical Engineering, Shanghai Jiao

Tong University, 800 Dongchuan Road, Shanghai 200240, China

E-mail: chuangxin.he@sjtu.edu.cn

H. Fu · C. He · Y. Liu

Gas Turbine Research Institute, Shanghai Jiao Tong University, 800 Dongchuan Road, Shanghai 200240, China 